ISSN: 0212-5374

DOI: http://dx.doi.org/10.14201/et201533183103

\title{
PERCEPCIONES DE DIRECTIVOS \\ Y PROFESORADO DE EDUCACIÓN FÍSICA SOBRE LAS COMPETENCIAS BÁSICAS
}

\section{Perceptions of managers and Physical Education teachers on Key Competencies}

\section{Perceptions des gestionnaires et des enseignants d'éducation physique sur les compétences de base}

David Hortigüela Alcalá*, Víctor Abellá García** y Ángel Pérez PueYo****

* Facultad de Humanidades y de Educación de Burgos. Correo-e: dhortiguela@ubu.es

**Facultad de Humanidades y de Educación de Burgos. Correo-e: vabella@ubu.es

**** Facultad de Ciencias de la actividad Física y del Deporte. Correo-e: angel.perez. pueyo@unileon.es

Recibido: 30-03-2014; Aceptado: 30-06-2014; Publicado: 30-04-2015 BIBLID [0212-5374 (2015) 33, 1; 83-103]

Ref. Bibl. DAVID HORTIGÜELA ALCALÁ, VÍCTOR ABELLÁ GARCÍA y ÁNGEL PÉREZ PUEYO. Percepciones de directivos y profesorado de Educación Física sobre las competencias básicas. Enseñanza \& Teaching, 33, 1-2015, 83-103.

RESUMEN: En este trabajo se presentan los resultados acerca de la percepción de los equipos directivos y los docentes de Educación Física (EF) sobre las competencias básicas (ССBB). La muestra utilizada fue de 30 centros de Educación Secundaria, administrando dos cuestionarios estructurados de manera categórica y validados por un grupo de expertos. Uno fue cumplimentado por los equipos directivos y otro por los profesores de Educación Física (EF). La metodología de trabajo ha sido mixta, realizando un estudio tanto cuantitativo como cualitativo. En el primer caso 
se ha llevado a cabo un análisis descriptivo que recoge frecuencias y porcentajes sobre cada una de las variables en las que se estructuran los cuestionarios. Respecto al análisis inferencial se han utilizado tablas de contingencia y $\chi^{2}$. En relación con la metodología cualitativa, se ha realizado un estudio de caso único en un centro escolar, empleándose como instrumentos de recogida de datos: entrevistas con el profesorado, diarios programados de observación de clases y grupos de discusión con el alumnado al finalizar el curso. La mayoría de los equipos directivos de los centros consideran necesario trabajar las CCBB, aunque muestran ciertas reticencias debido a: a) desconocimiento de diferentes propuestas de implantación, b) la escasa concienciación de los docentes respecto a su necesidad y c) la creencia generalizada del excesivo tiempo requerido. Los profesores de EF afirman que el trabajo de las СCBB en el aula puede generar un mayor aprendizaje del alumno y coherencia en la evaluación del proceso, pero queda patente la falta de organización y programación en el área para poder conseguir este fin.

Palabras clave: competencias básicas; evaluación formativa; aprendizaje significativo; programación didáctica; percepción docente; equipos directivos; profesor de educación física.

SUMMARY: Results on the perception of the management teams and teachers of Physical Education (PE) on Key Competencies (KC) are presented in this paper. The sample used was 30 secondary schools, administering two categorically structured questionnaires and validated by an expert group. One was completed by the management teams and another for teachers of Physical Education (PE). The working methodology has been mixed, making a quantitative and qualitative study. In the first case we have carried out a descriptive analysis that includes frequencies and percentages of each of the variables in the questionnaires are structured. Regarding the inferential analyzes were used contingency tables and $\chi^{2}$. In connection with the qualitative methodology, has conducted a single case study in a school, using as instruments of data collection: interviews with teachers, daily scheduled class observation and discussion groups with students after the course. Most management teams of schools considered necessary to work the KC but show some reluctance due to: a) lack of different proposals for implementation, b) low awareness of teachers about their need and, c) the widespread belief excessive time required. PE teachers claim that the work of the KC in the classroom can generate greater student learning and consistency in the assessment process, but it is clear the lack of organization and planning in the area to achieve this end.

Key words: key competencies; formative assessment; significative learning; teacher perception; management teams; physical education teacher.

RÉSUMÉ: Résultats sur la perception des équipes et des enseignants de l'éducation physique (EP) sur la gestion des compétences de base (CCBB) sont présentés dans le présent recherche. L'échantillon utilisé était de 30 écoles secondaires, deux questionnaires catégoriquement structurés et validés par un groupe d'experts. 
L'un a été complété par les équipes de gestion et une autre pour les enseignants de l'éducation physique (EP). La méthodologie de travail a été mélangé, faire une étude quantitative et qualitative. Dans le premier cas, nous avons effectué une analyse descriptive qui comprend les fréquences et les pourcentages de chacune des variables dans les questionnaires sont structurés. En ce qui concerne les analyses d'inférence ont été utilisés tableaux de contingence et $\chi^{2}$. En ce qui concerne la méthodologie qualitative, il ya eu une seule étude de cas dans une école, à l'aide d'instruments de collecte de données: entrevues avec les professeurs, les groupes d'observation de la classe et de discussion réguliers quotidiens avec les élèves après les cours. La plupart des équipes de gestion des écoles considérent comme nécessaires pour travailler les СCBB mais montrent une certaine réticence en raison de: a) l'absence de propositions différentes pour la mise en ouvre, b) la faible sensibilisation des enseignants au sujet de leur nécessité et, c) la croyance répandue temps excessif nécessaire. Professeurs d'éducation physique affirment que le travail du CCBB dans la salle de classe peut générer plus de l'apprentissage des élèves et de la cohérence dans le processus d'évaluation, mais il est clair que le manque d'organisation et de planification dans le domaine pour atteindre cette fin.

Mots clés: compétences clés; l'évaluation formative; l'apprentissage significatif; la perception des enseignants; équipes de gestion; professeur d'éducation physique.

\section{INTRODUCCIÓN}

Las diferentes investigaciones desarrolladas en marcos internacionales y europeos como el Informe Delors (Unesco, 1996), el Proyecto DeSeCo (ocDE, 2006), el Informe PISA (OCDE, 2009) o las Recomendaciones del Parlamento Europeo y del Consejo en materia de convergencia educativa (Consejo Europeo, 2000, 2001, 2002a, 2002b) han generado la necesidad de incorporar y definir un nuevo término denominado competencias clave (key competences). De entre las diferentes definiciones de estos organismos, la Comisión Europea define competencia clave como "una combinación de destrezas, conocimientos, aptitudes y actitudes, y a la inclusión de la disposición para aprender además del saber cómo" (Comisión Europea, 2004: 4); debiendo ser entendidas como "un paquete multifuncional y transferible de conocimientos, destrezas y actitudes que todos los individuos necesitan para su realización y desarrollo personal, inclusión y empleo" (Comisión Europea, 2004: 7).

Posteriormente, en un informe de progresos elaborado por el grupo de expertos designado por la Comisión Europea se establecieron ocho dominios de las competencias clave que posteriormente fueron ratificados en la Recomendación del Parlamento Europeo y del Consejo, de 18 de diciembre de 2006, sobre las competencias clave para el aprendizaje permanente.

La Ley Orgánica de Educación española, LOE 2/2006, determina que las Competencias Básicas (CCBB) han sido consideradas como un nuevo elemento curricular, que junto a los objetivos, contenidos, criterios de evaluación y métodos pedagógicos conforman los elementos del currículo (LOE, 2006: art. 6.1). En el Preámbulo de la actual Ley educativa se establece que el elemento del currículo 
que más interés reviste son las CCBB, "por cuanto debe permitir caracterizar de manera precisa la formación que deben recibir los estudiantes. Además, a ellas deben contribuir todas las materias, correspondiendo a la Administración ofertar el aprendizaje necesario para su adquisición" (LOE, 2006: art. 5.4).

Son muchas las propuestas existentes en la bibliografía que reflejan formas para contribuir a las ccBB (Blázquez, 2009; Díaz Hernando, 2008; Escamilla, 2008; Gitomer, Brown y Bonett, 2011; Grubb, 2012; Lleixá, 2007; Moya y Luengo, 2009; Pérez-Pueyo y Casanova, 2010; Proyecto Atántida, 2013; Polo, 2010; Sarramona, 2004; Sebastiani, Blázquez y Borrachina, 2009; Zabala y Arnau, 2007). Sin embargo, existe un desconcierto generalizado sobre cómo establecer en los centros educativos propuestas factibles y coherentes que permitan su desarrollo (Méndez, Mañana y Sierra, 2012). Esta falta de análisis real añade un ingrediente más a la motivación de este estudio, que conlleva la comprobación del verdadero trabajo de las CCBB que se realiza en los centros educativos. Aunque la actual Ley Educativa establece que todas las materias deben contribuir a la adquisición de las CCBB, no se establece ningún modelo oficial ni recomendado de desarrollo, programación y evaluación de las mismas.

Es necesario plantearse entonces algunas cuestiones como la causalidad de este hecho, cuál es el proceso que lleva a cabo cada centro o la necesidad de implantar una serie de criterios comunes de contribución a las competencias. Lo que es evidente es que la importancia que se otorga a las CCBB en el marco educativo actual es elevada, siendo el Estado el responsable de fijar programas de cooperación territorial que sirvan para reforzar las mismas (LOE, 2006: art. 9). Respecto a la propuesta de la LOMCE, las CCBB siguen teniendo un papel relevante y fundamental, considerándose como esos conocimientos básicos que tiene que adquirir el alumnado al finalizar la etapa obligatoria, en base a unas demandas, cada vez más específicas, del Marco Europeo. Sin embargo, actualmente, esta futura Ley no concreta, ni matiza ni incorpora nuevos planteamientos metodológicos en cuanto a su contribución. Si atendemos al RD 216/2014 por el que se establece el Currículo Básico de la Educación Primaria, se observa una modificación en el concepto, ahora Competencias Clave, definidas como «aquellas que todas las personas precisan para su realización y desarrollo personal, así como para la ciudadanía activa, la inclusión social y el empleo" (p. 19350). También se especifica que estas competencias son 7 en lugar de 8, no cambiando su carácter transversal e integral.

Otro de los interrogantes que se plantean es saber cuál es el proceso más coherente y eficaz para poder implantar las ССBB en los centros educativos, existiendo en la bibliografía modelos tanto inductivos como deductivos (Pérez-Pueyo, 2013a). La LOE (2006: art. 120) otorga a los centros autonomía pedagógica para concretar la necesaria adecuación a las características del contexto y del alumnado. Y es a partir de esta capacidad de concreción por la que estos deben interpretar el concepto de las CCBB, seleccionar una propuesta, desarrollar la integración de estas desde las programaciones didácticas hasta su aplicación a través de las programaciones de aula (Vermeulen et al., 2012). 
Esta falta de unanimidad a la hora de implantar en los centros un elemento curricular con tanta trascendencia a nivel educativo es una de las razones que justifican este estudio, analizando el tratamiento real que se realiza en el día a día. La percepción que tiene el docente es algo relevante en su contribución, más si cabe del profesorado de EF puesto que es habitual que les cueste comprender la razón de no contar con una competencia motriz (Méndez y López, 2009).

Tras las propuestas de autores como Lozano et al. (2012) o las investigaciones de Heras y Pérez-Pueyo (2012) y Pérez-Pueyo (2013b), basadas en el análisis de las propuestas más importantes en relación a una serie de criterios de valoración de las mismas, se establece que el enfoque más coherente es aquel en el que las СсBB deben emanar desde las intenciones educativas del centro, partiendo tanto de las características particulares que presente el mismo como de las intenciones que se pretenden.

Por más propuestas y planteamientos que se establezcan desde la Administración, no mejorará la contribución real que se haga en los centros educativos hasta que no exista una formación a los docentes, homogénea, progresiva y práctica (Moya y Luengo, 2009). Así, autores como Moya y Luengo (2009) afirman que el marco curricular es la referencia de donde deben extraerse las propuestas prácticas de actuación en el aula, siendo necesario en los centros plasmar un proyecto general de contribución consistente y aplicable por parte de los docentes. Es esta conexión entre lo curricular y la práctica diaria en los centros educativos lo que permitirá analizar y valorar con garantías el trabajo real de CCBB que se está llevando a cabo.

Para contribuir a las CCBB, además de tener un conocimiento y manejo exhaustivo de los elementos curriculares, se hacen fundamentales la organización y el funcionamiento de los centros, la participación del alumnado en los proyectos y una estructura de actuación común (Pharo y Bridle, 2012). Sin embargo, existen otros factores asociados y no menos importantes como el uso que se haga de la acción tutorial, el funcionamiento de la biblioteca, la relación con los padres y la planificación de actividades complementarias y extraescolares. Todo ello debe ser analizado por los centros educativos, para en función de sus características poder llevar a cabo un análisis de la documentación administrativa correspondiente y así implantar unas propuestas de trabajo en el aula más acordes (Pepper, 2011).

Por todo ello, en este estudio se presentan los proyectos en los que están sumidos los centros de secundaria y los resultados de su trabajo, exponiendo las conclusiones que establecen tanto los miembros de los equipos directivos como los docentes de EF. Estos datos son contrastados con el tipo de actuaciones que realizan los docentes en sus clases, utilizándose diferentes instrumentos para el registro de datos. Este tipo de análisis se realizará atendiendo a un modelo deductivo y multidisciplinar, analizando la contribución a las CCBB de manera integral y globalizada. 


\section{OBJETIVOS}

Los objetivos que se plantean en la investigación son:

1. Conocer el grado de relación existente entre la tipología y las características de los centros y las consideraciones de contribución a las CCBB por parte del equipo directivo.

2. Analizar cuál es el trabajo real de ссвB que se lleva a cabo en los centros educativos, relacionándolo con la percepción que tiene el docente sobre la inclusión de las mismas en el aula.

3. Observar la planificación existente en las tareas docentes relacionadas con las CCBB en la EF escolar, analizando el grado de coherencia existente entre lo reflejado en la Programación Didáctica (PD) y el desarrollo de las clases.

\section{MATERIALES Y MÉTODO}

\subsection{Muestra}

La población del estudio está compuesta por la totalidad de los centros de la capital de Burgos que imparten en sus enseñanzas Eso, un total de 30. De toda la muestra un $26,7 \%$ tiene carácter público, un $70 \%$ concertado y un $3,3 \%$ es de carácter privado.

Cada centro ha sido categorizado en función de su tipología, las etapas en las que se estructura, el rango de alumnos que tiene, el número de docentes y, en concreto, cuántos componen el departamento de EF.

\subsection{Análisis empleados}

En esta investigación se ha desarrollado una metodología mixta, en la que por un lado se ha utilizado un análisis estadístico mediante el paquete estadístico SPSS 18.0 y, por otro, un análisis cualitativo a través del programa de computación Weft-QDA. En cuanto a la estadística, se han realizado Tablas de Contingencia y Chi cuadrado, determinando la existencia de relación entre variables categóricas recogidas en cada uno de los ítems de los que constan los cuestionarios. El análisis cualitativo se realizará a partir de la recogida estructurada de la información de los estudiantes en base a las 4 categorías creadas a través de un estudio de caso en un centro en concreto. Posteriormente se ha realizado un análisis comparado de los 2 tipos de análisis a partir de la triangulación de datos.

\subsection{Instrumentos}

En base al tipo de metodología empleada en el estudio, se han diseñado diferentes instrumentos para la recogida de datos, tanto cuantitativos como cualitativos, estableciéndose categorías comunes según las variables asignadas. 


\subsubsection{Cuantitativos}

Se utilizan dos tipos de cuestionarios, ambos validados por un grupo de expertos. El primero de ellos fue dirigido al equipo directivo, y fundamentalmente evaluaba el grado de propuestas, implantación y seguimiento de CCBB que tenía el centro. El segundo se basa en la percepción que el docente de EF tiene sobre el trabajo de CCBB en el área y cómo desde el departamento se contribuía a la misma.

Se ha realizado el análisis de fiabilidad de cada uno de los cuestionarios a través del coeficiente alfa de Cronbach, obteniendo un resultado de 0,949 en el primero y 0,94 en el segundo.

El cuestionario final que cumplimentó el equipo directivo se compuso de 18 ítems, mientras que el dirigido a los jefes de departamento de EF tenía 15. Todos ellos responden a cuestiones categorizadas en una escala tipo Likert con valores de 1 a 5 (1 "no/nada", 2 "poco", 3 "bastante", 4 "mucho", 5 "no sabe"). La estructura del primer cuestionario se divide en 2 categorías: 1. "Importancia de las CCBB para el trabajo de centro" (10 cuestiones); 2. "Procesos de implantación metodológicos y evaluación aplicable al trabajo de CCBB" (8 cuestiones). El segundo cuestionario cumplimentado por los jefes de departamento de EF también se divide en 2 categorías: 1. "Implantación de las CCBB en las Programaciones didácticas y en el aula" (10 cuestiones), 2. "Estructura y organización para trabajar las CCBB en el área de EF” (5 cuestiones).

\subsubsection{Cualitativos}

Tres fueron los instrumentos que se emplearon para la obtención de datos y todos ellos enmarcados bajo el desarrollo de la técnica del estudio de caso que se llevó a cabo en el curso escolar 2012-2013.

El primer instrumento que se utilizó fue una entrevista inicial abierta realizada a cada uno de los 3 profesores de EF y en la que se pretendía obtener información sobre la percepción y el trabajo de las CCBB llevado a cabo en el día a día del aula. Cinco fueron las cuestiones generales que se plantearon a cada uno de los docentes sobre la concepción práctica de este elemento curricular, a partir de las cuales derivaron otras adyacentes: 1. ¿Cómo crees que contribuyes a las ccbb en el aula? ¿Podrias poner varios ejemplos concretos? 2. ¿Consideras que el área de la EF tiene una labor fundamental para este fin? ¿Diferente a otras materias? ¿Por qué? 3. ¿La metodología es un aspecto relevante y decisivo para su contribución? ¿Podrías poner un ejemplo? 4. ¿Es importante la relación con docentes de otras materias? ¿Lo haces? 5. ¿Crees que a través de su implantación en el aula el alumnado se implica más hacia el aprendizaje? ¿Mejora su relación interpersonal?

El segundo instrumento que se utiliza es un diario de observación, en el que mediante el análisis de las clases propias y de las de los compañeros en los 4 cursos de secundaria se registran 7 variables a partir de los diferentes agentes que interceden e influyen en el aula. Es un instrumento de seguimiento muy sencillo en el que diariamente se recogen: 1. Objetivos de la sesión; 2. Actividades; 
DAVID HORTIGÜELA ALCALÁ, VÍCTOR ABELLÁ GARCÍA Y ÁNGEL PÉREZ PUEYO PERCEPCIONES DE DIRECTIVOS Y PROFESORADO DE EDUCACIÓN FÍSICA...

3. Explicación de la sesión al alumno por parte del docente; 4. Grado de coherencia de los contenidos abordados; 5. Relación de trabajo interdisciplinar; 6. Vinculación con otro tipo de aprendizajes; 7. Reflexión/evaluación.

Finalmente y como cierre del curso escolar, en función de todo el proceso de seguimiento llevado a cabo, se realiza un grupo de discusión para cada uno de los 4 cursos con los que hemos trabajado, $1 .^{\circ}, 2 .^{\circ}, 3 .^{\circ}$ y $4 .^{\circ}$. Este se realiza en una sesión final para cada una de las clases, y se plantean las siguientes preguntas: 1. ¿Es la EF diferente al resto de materias? ¿Por qué? 2. ¿La EF influye en que fuera del aula realicéis más actividad física? ¿Por qué? 3. ¿Consideráis importante trabajar otros aspectos además de lo motriz (actividades orales, tareas de indagación, razonamiento)? ¿Por qué? 4. ¿Qué he aprendido este curso en EF? ¿Cuáles son las actividades en las que más aprendo?

Toda la información ha sido procesada por el programa de computación WeftQDA, agrupándose en cuatro categorías. Cada categoría integra una serie de variables de análisis:

1. En relación a la competencia motriz: vinculación con otras capacidades, percepción de éxito del alumnado, existencia de aprendizaje y utilización de test físicos.

2. Disparidad de criterios entre docentes: desacuerdo de opiniones en el departamento, coherencia con el planteamiento de contribución a las CCBB, grado en el que se aborda lo establecido en la Programación Didáctica, comentarios que derivan de la actuación diaria docente y tipo de materiales utilizados.

3. Grado de relación entre compañeros de aula: se introducen procesos de coevaluación y autoevaluación, existen metodologías que fomenten la variedad de agrupamientos, se realizan exposiciones orales en clase, se trabaja en grupo.

4. Percepción del alumno del trabajo realizado: grado de implicación en las tareas propuestas, preferencia para trabajar contenidos interdisciplinares, percepción sobre destrezas adquiridas.

\subsubsection{Codificación de instrumentos cualitativos}

A continuación se presenta un ejemplo de codificación para cada uno de los 3 instrumentos utilizados en el estudio de caso. EPEF $1 .^{\circ}$ : Entrevista profesor educación física $1 .^{\circ}$ curso, DP1. ${ }^{\circ}$ : Diario del profesor $1 .^{\circ}$ curso, GD1. ${ }^{\circ}:$ Grupo de discusión $1 .^{\text {er }}$ curso. Las dos primeras siglas servirán para dar nombre al instrumento empleado, mientras que el último dígito atenderá al curso en el que ha llevado a cabo. Este sistema categorial permite estructurar, regular y organizar la información obtenida, permitiendo hacer un tratamiento de los resultados más significativo. 


\section{ANÁLISIS DE RESULTADOS}

\subsection{Análisis cuantitativo}

\subsubsection{Cuestionario del equipo directivo}

Se observa que dos terceras partes de los equipos directivos consideran que el trabajo de las CCBB en los centros ayuda a crear una propia identidad del mismo (Tabla 1), mientras que solamente un 6,7\% dice que nada. En este sentido e independientemente de que los centros educativos establezcan propuestas de contribución a las competencias, más del $80 \%$ de la muestra afirma que el tratamiento real de este elemento curricular ayuda a la aplicación de los saberes adquiridos, existiendo por lo tanto un aprendizaje vinculado con la vida real.

TABLA 1

Porcentajes de respuestas de equipos directivos agrupados en la variable Importancia de las CCBB para el trabajo de centro

\begin{tabular}{|l|c|c|c|c|c|}
\hline \multicolumn{1}{|c|}{ PREGUNTAS } & \multicolumn{5}{|c|}{ PORCENTAJES DE RESPUESTAS } \\
\cline { 2 - 6 } & $\begin{array}{c}\text { NO/ } \\
\text { NADA } \\
(1)\end{array}$ & $\begin{array}{c}\text { POCO } \\
(2)\end{array}$ & $\begin{array}{c}\text { BASTANTE } \\
(3)\end{array}$ & $\begin{array}{c}\text { MUCHO } \\
(4)\end{array}$ & $\begin{array}{c}\text { NO SABE } \\
(5)\end{array}$ \\
\hline $\begin{array}{l}\text { Las CCBB contribuyen a la creación de } \\
\text { identidad de centro }\end{array}$ & 6,7 & 30 & 30 & 33,3 & 0 \\
\hline $\begin{array}{l}\text { Las CCBB facilitan la aplicación de los } \\
\text { saberes adquiridos }\end{array}$ & 3,3 & 13,3 & 53,3 & 30 & 0 \\
\hline $\begin{array}{l}\text { CCBB se vinculan con trabajo } \\
\text { interdisciplinar entre áreas }\end{array}$ & 3,3 & 46,7 & 40 & 10 & 0 \\
\hline $\begin{array}{l}\text { Si ha coordinado el tratamiento de las } \\
\text { CCBB por cursos }\end{array}$ & 3,3 & 26,7 & 60 & 10 & 0 \\
\hline $\begin{array}{l}\text { Trasladar el trabajo de CCBB a los } \\
\text { jefes de departamento }\end{array}$ & 6,7 & 33,3 & 43,3 & 16,7 & 0 \\
\hline $\begin{array}{l}\text { Metodologías que vinculen las CCBB } \\
\text { con realidad práctica }\end{array}$ & 3,3 & 6,7 & 26,7 & 60 & 3,3 \\
\hline $\begin{array}{l}\text { Tratamiento de las CCBB fomenta } \\
\text { diálogo y participación }\end{array}$ & 0 & 16,7 & 30 & 50,3 & 0 \\
\hline $\begin{array}{l}\text { Favorecen visión más amplia del } \\
\text { concepto de aprendizaje }\end{array}$ & 0 & 20 & 40 & 40 & 0 \\
\hline $\begin{array}{l}\text { Es necesaria una secuenciación de las } \\
\text { CCBB por cursos }\end{array}$ & 0 & 16,7 & 56,7 & 23,3 & 3,3 \\
\hline $\begin{array}{l}\text { Relevancia de las CCBB para el } \\
\text { aprendizaje }\end{array}$ & 0 & 6,7 & 60 & 33,3 & 0 \\
\hline
\end{tabular}


A pesar de que casi un 40\% de los equipos directivos admite no transmitir información relacionada con las competencias a los jefes de cada departamento para poder desarrollar estrategias en el aula, admiten la importancia que tiene el tratamiento de este elemento curricular para el fomento del diálogo y de la participación.

TABLA 2

Frecuencia de respuestas de los equipos directivos agrupados en la variable Procesos de implantación metodológicos y evaluación aplicable al trabajo de CCBB

\begin{tabular}{|l|c|c|c|c|c|}
\hline \multicolumn{1}{|c|}{ PREGUNTAS } & \multicolumn{5}{|c|}{ PORCENTAJES DE RESPUESTAS } \\
\cline { 2 - 7 } & $\begin{array}{c}\text { NO/NADA } \\
(1)\end{array}$ & $\begin{array}{c}\text { POCO } \\
(2)\end{array}$ & $\begin{array}{c}\text { BASTANTE } \\
(3)\end{array}$ & $\begin{array}{c}\text { MUCHO } \\
(4)\end{array}$ & $\begin{array}{c}\text { NO SABE } \\
(5)\end{array}$ \\
\hline $\begin{array}{l}\text { Permiten mayor aprendizaje del } \\
\text { profesor en el proceso }\end{array}$ & 3,3 & 23,3 & 50 & 23,3 & 0 \\
\hline $\begin{array}{l}\text { Se abordan propuestas concretas de } \\
\text { trabajo }\end{array}$ & 10 & 36,7 & 36,7 & 16,7 & 0 \\
\hline $\begin{array}{l}\text { Es necesario que hagan al alumno } \\
\text { trabajar autónomamente }\end{array}$ & 3,3 & 6,7 & 40 & 50 & 0 \\
\hline $\begin{array}{l}\text { Es necesario que el alumno fomente } \\
\text { su reflexión crítica }\end{array}$ & 3,3 & 6,7 & 43,3 & 43,3 & 3,3 \\
\hline $\begin{array}{l}\text { Permiten que el alumno relacione e } \\
\text { interprete conceptos }\end{array}$ & 0 & 13,3 & 23,3 & 63,3 & 0 \\
\hline $\begin{array}{l}\text { Permiten una evaluación más } \\
\text { consensuada y rigurosa }\end{array}$ & 3,3 & 26,7 & 46,7 & 20 & 3,3 \\
\hline $\begin{array}{l}\text { Permiten mayor variedad de } \\
\text { alternativas en la evaluación }\end{array}$ & 0 & 16,7 & 50 & 33,3 & 0 \\
\hline $\begin{array}{l}\text { Permite que el alumno evalúe el } \\
\text { trabajo de los demás }\end{array}$ & 6,7 & 26,7 & 43,3 & 20 & 3,3 \\
\hline
\end{tabular}

Tal y como podemos observar en la Tabla 2 de nuevo se constata la importancia que se otorga al tratamiento de las CCBB con el fin de que el alumno fomente su reflexión crítica. En este sentido, y alcanzando también unos porcentajes elevados de respuesta, el 73,3\% de los equipos de gobierno considera que abordar estos procesos permite un mayor aprendizaje del profesorado a lo largo del proceso. En este sentido y de manera categórica, un 90\% afirma que el trabajo de competencias tiene que servir para permitir al alumno trabajar de una manera autónoma, fomentando también su reflexión crítica.

El ítem que más controversia genera en la muestra, obteniendo unos resultados más dispares, es el que trata de abordar propuestas concretas de trabajo, debido al escaso tratamiento práctico que existe de las competencias. 
TABLA 3

Tablas de Contingencia y $\chi^{2}$ de ítems extraídos del cuestionario del equipo directivo

\begin{tabular}{|l|c|c|c|}
\hline \multicolumn{1}{|c|}{ RELACIÓN ENTRE VARIABLES } & \multicolumn{2}{c|}{ CHI-SQUARE TEST } \\
\cline { 2 - 4 } & $\chi^{2}$ & $D F$ & $P$ \\
\hline $\begin{array}{l}\text { Creación de identidad del centro/Mayor aprendizaje del profesorado } \\
\text { en el proceso }\end{array}$ & 18,26 & 9 & .032 \\
\hline $\begin{array}{l}\text { Aplicación de los saberes adquiridos/relaciona conceptos e } \\
\text { interpreta }\end{array}$ & 4,81 & 6 & .569 \\
\hline $\begin{array}{l}\text { Hagan al alumno trabajar autónomamente/fomente su reflexión } \\
\text { crítica }\end{array}$ & 58,25 & 12 & .000 \\
\hline Tratamiento de las cCBB por cursos/Es necesaria su secuenciación & 4,02 & 9 & .910 \\
\hline $\begin{array}{l}\text { Mayor variedad de alternativas en la evaluación/el alumno evalúe } \\
\text { a los demás }\end{array}$ & 14,47 & 8 & .070 \\
\hline $\begin{array}{l}\text { Favorecen visión más amplia de aprendizaje/Relevancia para el } \\
\text { aprendizaje }\end{array}$ & 13,67 & 4 & .008 \\
\hline CCBB con realidad práctica/CCBB fomenta diálogo y participación & 6,54 & 6 & .365 \\
\hline $\begin{array}{l}\text { Fomento de reflexión crítica/Aprendizaje vinculado con realidad } \\
\text { práctica }\end{array}$ & 79,54 & 16 & .000 \\
\hline
\end{tabular}

En la Tabla 3 se observa cómo se obtiene la significatividad en la relación de 3 pares de ítems. Todos ellos constatan la coherencia mostrada en el análisis descriptivo, ya que las cuestiones se vinculan con el trabajo autónomo del alumno, el fomento de la reflexión crítica y el mayor aprendizaje. Llama la atención la no significatividad entre el tratamiento de las CCBB por cursos y la necesidad de secuenciación $\left(\chi_{(30)}^{2}=4,025, p=, 910\right)$, o la referente a la mayor variedad de alternativas en la evaluación frente a que el alumno evalúe a los demás, no alcanzando esta porcentajes muy favorables.

\subsubsection{Cuestionario del docente de EF}

En líneas generales, los resultados mostrados en la Tabla 4 abarcan unos rangos de respuesta más dispares que en el caso de los cuestionarios cumplimentados por los equipos directivos. En relación al ámbito curricular, un amplio porcentaje de la nuestra afirma reflejar las CCBB en la PD, relacionando estas con los CE correspondientes. Destaca también la unanimidad que muestran los docentes a la hora de destacar que no solamente hay que contribuir al comienzo y al final de la etapa, siendo necesaria la determinación de ítems comunes para todas las áreas. 
TABLA 4

Porcentajes de respuestas de los docentes de EF agrupados en la variable Implantación de las CCBB en las PD y en el aula

\begin{tabular}{|l|c|c|c|c|c|}
\hline \multirow{2}{*}{ PREGUNTAS } & \multicolumn{5}{|c|}{ PORCENTAJES DE RESPUESTAS } \\
\cline { 2 - 6 } & $\begin{array}{c}\text { NO/NADA } \\
(1)\end{array}$ & $\begin{array}{c}\text { POCO } \\
(2)\end{array}$ & $\begin{array}{c}\text { BASTANTE } \\
(3)\end{array}$ & $\begin{array}{c}\text { MUCHO } \\
(4)\end{array}$ & $\begin{array}{c}\text { NO SABE } \\
(5)\end{array}$ \\
\hline $\begin{array}{l}\text { Trabajo conjunto entre } \\
\text { departamentos }\end{array}$ & 16,7 & 36,7 & 33,3 & 13,3 & 0 \\
\hline $\begin{array}{l}\text { Refleja en la PD la contribución a las } \\
\text { CCBB }\end{array}$ & 0 & 16,7 & 33,3 & 50 & 0 \\
\hline $\begin{array}{l}\text { Vincular los criterios de evaluación } \\
\text { (CE) con las CCBB }\end{array}$ & 3,3 & 23,3 & 33,3 & 40 & 0 \\
\hline $\begin{array}{l}\text { Permiten la variedad de alternativas } \\
\text { en el aula }\end{array}$ & 3,3 & 16,7 & 46,7 & 33,3 & 0 \\
\hline $\begin{array}{l}\text { Necesidad de evaluación formativa } \\
\text { durante el curso }\end{array}$ & 0 & 16,7 & 46,7 & 36,7 & 0 \\
\hline $\begin{array}{l}\text { Desde el departamento nacen las } \\
\text { propuestas }\end{array}$ & 0 & 20 & 50 & 30 & 0 \\
\hline Desde un área se contribuya a todas & 13,3 & 30 & 23,3 & 33,3 & 0 \\
\hline $\begin{array}{l}\text { Solo se contribuye en 1. }{ }^{\circ} \text { y 4. } .^{\circ} \text { por } \\
\text { cambio de la etapa }\end{array}$ & 23,3 & 53,3 & 13,3 & 6,7 & 3,3 \\
\hline Ítems comunes para todas las áreas & 0 & 10 & 53,3 & 33,3 & 3,3 \\
\hline $\begin{array}{l}\text { Plantea juicios subjetivos que } \\
\text { dificultan la evaluación }\end{array}$ & 6,7 & 40 & 33,3 & 10 & 10 \\
\hline
\end{tabular}

TABLA 5

Frecuencias de respuestas de los docentes de EF en la variable Estructura y organización para trabajar las CCBB en el área de EF

\begin{tabular}{|l|c|c|c|c|c|}
\hline \multirow{2}{*}{ PREGUNTAS } & \multicolumn{5}{|c|}{ PORCENTAJES DE RESPUESTAS } \\
\cline { 2 - 6 } & $\begin{array}{c}\text { NO/NADA } \\
(1)\end{array}$ & $\begin{array}{c}\text { POCO } \\
(2)\end{array}$ & $\begin{array}{c}\text { BASTANTE } \\
(3)\end{array}$ & $\begin{array}{c}\text { MUCHO } \\
(4)\end{array}$ & $\begin{array}{c}\text { NO SABE } \\
(5)\end{array}$ \\
\hline $\begin{array}{l}\text { Reuniones de departamento para } \\
\text { evaluar las CCBB }\end{array}$ & 10 & 36,7 & 50 & 3,3 & 0 \\
\hline $\begin{array}{l}\text { Incorporación de una competencia } \\
\text { motriz }\end{array}$ & 0 & 10 & 33,3 & 50 & 6,7 \\
\hline $\begin{array}{l}\text { Procedimientos de evaluación } \\
\text { variados y adecuados }\end{array}$ & 0 & 0 & 30 & 70 & 0 \\
\hline Metodologías cooperativas en el aula & 0 & 13,3 & 43,3 & 43,3 & 0 \\
\hline $\begin{array}{l}\text { La formación docente es } \\
\text { fundamental para trabajar las CCBB }\end{array}$ & 0 & 6,7 & 56,7 & 36,7 & 0 \\
\hline
\end{tabular}


En los análisis mostrados en la Tabla 5 existen algunas controversias; si bien más de un 80\% afirma la necesidad de incorporar una competencia motriz a las 8 ya existentes, casi la mitad de los docentes de EF no aborda la temática de las CCBB en las reuniones de departamento.

En relación al plano metodológico, la muestra determina la necesidad de utilizar una variedad de instrumentos de evaluación, implantar metodologías basadas en el aprendizaje cooperativo y sobre todo llevar a cabo una formación docente para su desarrollo.

Al igual que sucediera en los análisis realizados con el cuestionario dirigido a los equipos directivos, se alcanza la significatividad en 3 pares de ítems (Tabla 6). El primero se encuentra relacionado con la evaluación formativa y la formación del docente $\left(\chi_{(30)}^{2}=11,136, p=.012\right)$, y los otros 2 tienen por denominador común el uso de las metodología cooperativas, vinculándose tanto con la evaluación $\left(\chi_{(30)}^{2}=2,985, p=.0 .25\right)$, como con la incorporación de una competencia motriz $\left(\chi_{(30)}^{2}=6,322, p=.707\right)$.

Sin embargo, no existe significatividad entre los ítems del trabajo común entre departamentos y los juicios subjetivos que dificulten la evaluación, siendo este último un aspecto que la muestra considera como problemática.

TABLA 6

Tablas de Contingencia y $\chi^{2}$ de ítems extraídos del cuestionario del profesorado de EF

\begin{tabular}{|l|c|c|c|}
\hline \multirow{2}{*}{ RELACIÓN ENTRE VARIABLEs } & \multicolumn{2}{|c|}{ CHI-SQUARE TEST } \\
\cline { 2 - 4 } & $\chi^{2}$ & $D F$ & $P$ \\
\hline $\begin{array}{l}\text { Variedad de alternativas en el aula/Necesidad de evaluación } \\
\text { formativa en curso }\end{array}$ & 11,07 & 6 & .086 \\
\hline $\begin{array}{l}\text { Desde el departamento nacen las propuestas/Ítems comunes } \\
\text { para todas las áreas }\end{array}$ & 7,21 & 6 & .301 \\
\hline $\begin{array}{l}\text { Evaluación formativa durante el curso/La formación docente } \\
\text { es fundamental }\end{array}$ & 11,14 & 4 & .012 \\
\hline $\begin{array}{l}\text { Vincular los CE con las CCBB/Incorporación de una } \\
\text { competencia motriz }\end{array}$ & 6,32 & 9 & .707 \\
\hline $\begin{array}{l}\text { Trabajo común entre departamentos/juicios subjetivos que } \\
\text { dificulten la evaluación }\end{array}$ & 11,61 & 12 & .477 \\
\hline $\begin{array}{l}\text { Incorporación de una competencia motriz/metodologías } \\
\text { cooperativas de aula }\end{array}$ & 11,02 & 6 & .048 \\
\hline $\begin{array}{l}\text { Desde el área de EF se puede contribuir/sólo en } 1 .^{\circ} \text { y } 4 .^{\circ} \text { por } \\
\text { cambio de etapa }\end{array}$ & 13,12 & 8 & .108 \\
\hline $\begin{array}{l}\text { Procedimientos de evaluación variados y adecuados/ } \\
\text { metodologías cooperativas }\end{array}$ & 2,98 & 2 & .025 \\
\hline
\end{tabular}




\subsection{Análisis cualitativo}

Para recoger los datos obtenidos en el estudio de caso único realizado a lo largo de un curso escolar, se han establecido cuatro categorías que atienden a la información extraída de cada uno de los instrumentos utilizados.

\subsubsection{En relación a la competencia motriz}

Se observa en la información recogida a lo largo del proceso como existe un trabajo de lo motriz verdaderamente dispar, siendo la metodología y el tipo de contenidos utilizados por el docente de EF muy diferentes para contribuir a las CCBB:

Independientemente de la contribución que tengamos que hacer a las Competencias Básicas, en la Educación Física lo más importante es que el alumno se mueva para alcanzar esa competencia motriz, cosa que el resto de asignaturas ni puede ni debe hacer [...] (EPEF4. $\left.{ }^{\circ}\right)$.

La Educación Física es algo más que el alumno se esté moviendo continuamente sin saber por qué, debemos estructurar los contenidos que vamos a trabajar, darles una coherencia y sobre todo preguntarnos qué es lo que el alumno aprende en nuestras clases [...], solamente pensando así, podremos ponernos de acuerdo para saber a qué CCBB debe contribuir el alumno (EPEF2..$^{\circ}$ ).

Como consecuencia, estos planteamientos se traducen en los aspectos prácticos desarrollados en el aula y que han sido observados en el análisis de las clases:

[...] Estamos a final del segundo trimestre y no se observa ninguna estructura en los contenidos de las UD's, los chicos juegan, se divierten, se les hacen varios test de condición física [...] pero no nunca se constata el aprendizaje del alumno (DP4. ${ }^{\circ}$ ). Todas las clases acaban con una reflexión final en la que todos los alumnos participan sobre lo acontecido [...], no todo lo que se trabaja es puramente motriz sino que las relaciones interpersonales y la inserción social son 2 aspectos que siempre se tienen en cuenta, se busca el logro grupal [...] (DP2. ${ }^{\circ}$.

Finalmente, es en los grupos de discusión donde observamos las valoraciones finales que realizan los alumnos tras haber cursado la asignatura, mostrando percepciones variadas:

Ha sido lo de siempre [...], los chicos jugando al fútbol, las chicas al voley y a los que menos nos gusta el deporte a pasarlo mal con las pruebas físicas (GD $4 .^{\circ}$ ).

Sin embargo, la motivación no siempre era baja aunque el trabajo se vinculara únicamente al ámbito motriz:

Para mí la "gimnasia" es la mejor asignatura, yo saco buenas notas siempre [...], [Investigador:] ¿Qué has aprendido? Aprender... mmmm, a saltar, a correr [...] $\left(\mathrm{GD} 4 .^{\circ}\right)^{\circ}$. 
Lo que sí que se observa es una motivación elevada hacia el curso en el que se han trabajado más aspectos que los únicamente motrices:

Ha sido muy diferente [...]. Hemos practicado una gran cantidad de deportes y tareas, hemos trabajado en equipo y hemos hecho muchas exposiciones orales (GD2. ${ }^{\circ}$ ). He conseguido hacer todo lo que proponía el profesor y nunca he tirado la toalla [...]. [Investigador:] ¿Estás más motivada? Mucho más, otros años he suspendido por no saltar mucho o no lanzar muy lejos el balón, y otras veces aprobaba y ¡no sabía muy bien por qué!

\subsubsection{Disparidad de criterios entre docentes}

Contrastando los datos observamos algunas incoherencias entre lo que se dice y lo que se hace, debido en gran manera a la falta de seguimiento de la PD establecida por parte del departamento y a la inexistencia de criterios comunes para contribuir a las competencias:

Entiendo que a las competencias básicas cuesta contribuir ahora en un comienzo [...]. No obstante yo procuro trabajar contenidos de otras asignaturas, variar la metodología, que el alumno busque información, trabaje en grupo... (EPEF3. ${ }^{\circ}$. Se vuelven a trabajar aspectos similares a los que se desarrollaron en el $2 .^{\circ}$ trimestre. Fundamentalmente aspectos deportivos donde el alumno tiene exámenes prácticos y teóricos (DP3. ${ }^{\circ}$.

No obstante y aun reconociendo que no es lo verdaderamente correcto, la linealidad en los planteamientos iniciales se lleva a cabo a lo largo de todo el curso:

Sobre todo lo que los chicos quieren es jugar, ya que vienen de primaria, se trabajen o no las competencias que dices [...]. La programación es algo que está ahí, pero sinceramente yo no la hago caso $\left(\operatorname{EPEF} 1 .^{\circ}\right)$. Estamos a final del primer trimestre y se observa la improvisación con la que actúa el profesor en relación a los contenidos [...], hoy fútbol, mañana correr... Esta desestructuración muestra una imposibilidad del trabajo coherente de las competencias (DP1. ${ }^{\circ}$ ).

\subsubsection{Grado de relación entre compañeros de aula}

Se observó el grado de relación interpersonal que tenían los alumnos a lo largo del curso, incidiendo en el tipo de actividades planteadas, agrupamientos, actitud del docente...

Los alumnos trabajan en grupo desde el comienzo [...]. No importa quien juegue mejor, sino que siempre el docente busca un logro grupal de la clase que hace que aumente la motivación (DP2..$^{\circ}$. Los alumnos se divierten pero existen muchas faltas de respeto entre compañeros [...], gastan bromas siempre a los mismos que no gustan a los demás, y que en mi opinión no son tolerables [...]. El docente no 
DAVID HORTIGÜELA ALCALÁ, VÍCTOR ABELLÁ GARCíA Y ÁNGEL PÉREZ PUEYO PERCEPCIONES DE DIRECTIVOS Y PROFESORADO DE EDUCACIÓN FÍSICA...

hace nada (DP1. ${ }^{\circ}$ ). Existen grupos bien diferenciados los cuales están siempre juntos y que ignoran al resto. El sentimiento de grupo y unanimidad es escaso (DP4. ${ }^{\circ}$ ).

Estos aspectos reflejados en el diario de clase repercuten lógicamente en la percepción del grupo:

Tenía mucho miedo a la hora de hacer algunas cosas [...]. Gracias al profe y a la clase he conseguido lo que creía que no era posible (DP2. ${ }^{\circ}$ ). A mí me gusta Educación Física, lo que pasa es que Roberto y Santi nunca paran de insultarme y el profe no les dice nada (DP1. ${ }^{\circ}$ ). En Educación Física paso buenos ratos con mis amigas [...] [Investigador:] ¿Trabajáis en grupo? En grupo, sí siempre con mis amigas (risas) $\left(\right.$ DP $\left.^{\circ} .^{\circ}\right)$.

\subsubsection{Percepción del alumno del trabajo realizado}

Estos aspectos quedan reflejados fundamentalmente en los grupos de discusión de cada uno de los cursos, no obstante en los diarios de clase también se observan muchas percepciones y valoraciones del alumnado.

Pablo lleva realizando la prueba de abdominales dos veces y no llega al 5 . Se le ve desesperado sobre todo porque no sabe lo que hacer para poder aprobar. El profesor únicamente le dice que tiene que repetirlo la semana que viene y esforzarse más... increíble (DP4. ${ }^{\circ}$ ). De nuevo el profesor ha realizado la reflexión final de la clase y las percepciones del alumno han sido muy buenas (DP2. ${ }^{\circ}$ ).

Hemos realizado un montón de pruebas físicas para mantenernos en forma [...], si se te da bien apruebas y si no suspendes. [Investigador:] ¿Consideráis eso injusto? $\left(\mathrm{GD} 4{ }^{\circ}\right)$. Bueno un poco, la verdad. Trabajar en grupos para mí ha sido lo mejor de la asignatura [...] nos ha servido para saber lo que hacen los demás y repartirnos mucho mejor el trabajo (GD2. ${ }^{\circ}$ ).

\section{DISCUSIÓN Y CONCLUSIONES}

En relación al primer objetivo planteado en el estudio ha quedado de manifiesto en la investigación la importancia que los equipos directivos de los centros otorgan a las CCBB como elemento curricular, teniendo tras de sí una vía de trabajo positiva que implica a una gran parte del profesorado. La perspectiva de trabajo hacia este elemento curricular en líneas generales es positiva, pero sin embargo los equipos directivos reconocen carecer de estrategias y procedimientos para desarrollar vías de trabajo coherentes que evalúen su contribución. En este sentido, Braun (2011) determina que esta percepción docente, si se combina con un trabajo colaborativo a largo plazo que permita el intercambio de experiencias, proyectará una formación más multidisciplinar y una estabilidad en los resultados. 
En relación a la existencia de propuestas de trabajo en los centros, hemos observado que la mayoría de la muestra presenta vías de actuación de manera independiente entre algunos docentes. Esto deriva en resultados más propios de "buenas intenciones" que en proyectos deductivos de carácter global (Heras y Pérez-Pueyo, 2012). Este hecho repercute en otro de los aspectos reflejados en el estudio, que es que la mayor parte de los docentes incluye el trabajo de las CCBB en las PD, pero sin existir un acuerdo en los aspectos comunes que se pueden abordar y trabajar conjuntamente. Este hecho, tal y como comentan Moya y Luengo (2009), provoca una concepción errónea en la contribución a las competencias, careciendo de sentido que se proyecte hacia aspectos no comunes en los mismos cursos.

Uno de los aspectos que más unanimidad ha mostrado la investigación ha sido la relación existente entre el trabajo de las competencias en los centros y la relación de estas con el desarrollo de metodologías que conexionen el aprendizaje con la realidad práctica. Pepper (2011) afirma que este aspecto es el fundamental y prioritario en el trabajo de las ССBB, debiendo vincular los objetivos de la educación hacia ese saber hacer en el día a día.

Es necesario vincular las competencias con una evaluación aplicada y coherente. En este sentido, la muestra refleja una variedad de respuestas acerca de este hecho, manifestando dudas razonables sobre si la contribución a las CCBB permite una evaluación más consensuada y rigurosa. En contra de esta percepción, Lozano et al. (2012) afirman que la forma de proceder en relación a las competencias debería delimitar acciones comunes para contribuir a aspectos concretos, algo que manifestaría un mayor consenso en la evaluación. Este hecho demuestra la falta de coherencia y unanimidad en cuanto a la determinación de una propuesta común por parte de los centros en la muestra estudiada.

Respecto al trabajo por competencias y la percepción del docente sobre la inclusión de las mismas en el aula, y habiendo quedado patente la vinculación que establecen los equipos directivos entre la contribución a las CCBB y el mayor aprendizaje que puede conllevar su desarrollo, en el presente estudio se ha demostrado la percepción favorable que tiene la muestra hacia el tratamiento de las competencias y su consecuente aumento de autonomía en el aprendizaje por parte del alumno. Además, este hecho se vincula con la necesidad de fomento de la reflexión crítica en cada una de las tareas abordadas. En este sentido, autores como Herts y Assumpció (2011) indican que en la actualidad uno de los denominadores comunes que plantea el trabajo de las CCBB es la predisposición favorable de los docentes a su desarrollo, asumiendo todas sus ventajas y beneficios y favoreciendo la motivación en el alumnado Carbonero, Martín-Antón, Román y Reoyo (2010). Sin embargo, este aspecto no se relaciona con el trabajo real y las herramientas de puesta en práctica que realicen los propios profesores.

Como respuesta al tercer objetivo del estudio y en relación a los docentes de EF y a la puesta en práctica de estrategias concretas en el aula que contribuyan a dicho elemento curricular, los resultados demuestran que los profesores consideran determinante abordar las competencias bajo un sistema de evaluación formativa, 
entendido como ese proceso de aprendizaje constante en el que el alumno aprende en el día a día y se le permite mejorar a lo largo del transcurso del curso, no sólo al final. Este aspecto, además de relacionarse con un mayor aprendizaje, se vincula con la necesidad de asociarlo al desarrollo de unos procedimientos de evaluación variados y adecuados en cada una de las Unidades Didácticas (UD). Algunos autores, como Gitomer, Brown y Bonett (2011), consideran que la clave de un aprendizaje efectivo del alumnado no se encuentra únicamente relacionado con el tratamiento de una gran cantidad de tareas, sino con el hecho de determinar objetivos comunes en función de los contenidos desarrollados. Además, Parrilla (1999) establece que este trabajo conjunto puede aportar un elevado componente innovador en el aula, reflexionando diariamente sobre la práctica.

En este sentido y en relación a la muestra, se ha observado como la desestructuración planteada en la PD del departamento de EF repercute en una incoherencia en el tratamiento de los contenidos por niveles, no estableciéndose progresiones coherentes ni aplicando en el aula lo acordado. Se corrobora que el planteamiento superficial de contribución a las ССBB que se refleja en la programación no tiene una traducción sólida en la práctica diaria.

Uno de los aspectos que más controversia ha generado desde la implantación de las с han solido reivindicar los docentes de EF. El presente estudio viene a reiterar esta idea, estableciendo como una carencia del currículo que el aspecto motriz no se relacione con este elemento curricular. En relación a la propia práctica y analizando los resultados del estudio de caso, se ha observado la metodología dispar que empleaba cada uno de los docentes del departamento, en varios casos incluso alejada de los propios objetivos marcados en la programación de aula. Este aspecto además de influir en la contribución a las CCBB, incide de manera directa en la reacción de los alumnos, provocando una reacción más negativa cuando la calificación se fundamentaba únicamente en la realización de los test físicos. En este hecho inciden autores como Standage Duda y Ntoumanis (2003), ya que establecen que la variedad en los contenidos de EF a lo largo de los cursos permite alcanzar una mayor motivación en el alumnado y un registro más concreto de su aprendizaje.

La metodología mixta de esta investigación ha permitido analizar la percepción de los equipos directivos y docentes de EF sobre la implantación y el trabajo de las CCBB, elemento curricular clave en el sistema educativo español. En futuras investigaciones relacionadas con este ámbito, se propone el aumento de la muestra o el análisis en concreto de centros que hayan incorporado propuestas de trabajo concretas.

Por ello, y a pesar de la predisposición positiva que tienen los equipos directivos de los centros de educación secundaria hacia la implantación de procesos coherentes de CCBB, es mucha la desinformación todavía existente. Este hecho repercute en que se planteen muy buenos trabajos por parte de docentes aislados, pero que no se integren dentro de la filosofía del ámbito de las competencias al 
carecer de objetivos comunes del centro. Es necesario por lo tanto que se definan por parte de la administración líneas y orientaciones concretas del trabajo de competencias en el centro, permitiendo también una formación permanente para toda la comunidad educativa

\section{REFERENCIAS BIBLIOGRÁFICAS}

Blázquez, D. (2009). Enseñar por Competencias en Educación Física. Barcelona: Inde.

Braun, M. P. (2011). Self-rated competences and future vocational success: a longitudinal study. Assessment of Vocational Competence in Higher Education, 36 (4), 417-427. http://dx.doi.org/10.1080/02602938.2010.534762

Carbonero, M. A.; Martín-Antón, L. J.; Román, L. M. y Reoyo, N. (2010). Efecto de un programa de entrenamiento al profesorado en la motivación, clima del aula y estrategias de aprendizaje de su alumnado. Revista Iberoamericana de Psicología y Salud, 1 (2), $117-138$.

CONSEJO DE LA UnIÓN EuROPea (2000). Informe del Consejo Europeo de Lisboa. Conclusiones Presidenciales. Recuperado de http://www.europarl.europa.eu/summits/lis1_es.htm.

Consejo de la Unión EuRopea (2001). Informe del Consejo Educación al Consejo Europeo sobre los futuros objetivos precisos de los sistemas de educación y formación.

http://europa.eu/legislation_summaries/education_training_youth/general_framework/c11049_es.htm.

Consejo de la Unión Europea (2002a). Informe del Consejo Europeo de Barcelona. Conclusiones Presidenciales.

http://www.consilium.europa.eu/ueDocs/cms_Data/docs/PressData/es/ec/70829.pdf.

Consejo de la Unión EuRopea (2002b). Programa de trabajo detallado para el seguimiento de los objetivos concretos de los sistemas de educación y formación de Europa. Diario Oficial de las Comunidades Europeas.

http://eurlex.europa.eu/LexUriServ/LexUriServ.do?uri=OJ:C:2002:142:0001:0022:ES:pdf.

Delors, A. J. (1996). La educación encierra un tesoro. Informe a la UNESCO de la Comisión Internacional sobre la Educación para el Siglo XxI. Madrid: Santillana.

Díaz-Hernando, C. (2008). La Competencia Social y Ciudadana en la LOE. Revista de la Asociación de Inspectores de Educación de España, 9 (2), 15-18.

Escamilla, A. (2008). Las CCBB: claves y propuestas para su desarrollo en los Centros. Barcelona: Graó.

Gitomer, D.; Brown, T. y Bonett, J. (2011). Useful signal or unnecessary obstacle? The role of basic skills tests in teacher preparation. Journal of Teacher Education, 62 (5), 431-445. http://dx.doi.org/10.1177/0022487111412785

Grubb, W. N. (2012). Basic Skills Education in Community Colleges. Kentucky: Routlegde.

Heras, C. y Pérez-Pueyo, A. (2012). Las competencias básicas en el marco del proyecto educativo europeo Comenius: Propuesta de secuenciación y desarrollo. Revista Española de Educación Física y Deportes, 398 (3), 35-58.

Herts, A. y Sempere, A. (2011). Nuevos modelos de formación para el trabajo por competencia. Actas del Congreso Estatal de Formación del Profesorado. Madrid, 15, 16 y 17 de junio de 2011. 
Lleixá, T. (2007). Educación física y competencias básicas: contribución del área a la adquisición de las competencias básicas del currículo. Tándem: Didáctica de la Educación Física, 23, 31-37.

LOE (2006). Ley Orgánica de Educación. http://www.boe.es/boe/dias/2006/05/04/pdfs/A17158-17207.pdf.

LOMCE (2014). Ley Orgánica para la Mejora de Calidad Educativa. http://www.boe.es/boe/dias/2013/12/10/pdfs/BOE-A-2013-12886.pdf

Lozano, A.; Boni, A.; Peris, J. y Hueso, A. (2012). Competencies in Higher Education: A critical Analysis from the capabilities approach. Journal of Philosophy of Education, 46 (1), 132-147. HTTP://DX.DOI.ORG/10.1111/J.1467-9752.2011.00839.X

Moya, J. y Luengo, F. (2009). Las competencias básicas en la práctica. Madrid: Proyecto Atlántida.

MEC (2007). PISA, 2006. Programa para la evaluación internacional de alumnos de la OCDE. Informe español. Centro de Publicaciones: Secretaría General Técnica.

Méndez, A. y López, T. (2009). Competencias básicas: sobre la exclusión de la competencia motriz y las aportaciones desde la Educación Física. Retos: Nuevas Tendencias en Educación Física, Deporte y Recreación, 16, 51-57.

Méndez, A.; Mañana, J. y Sierra, B. (2012). Necesidad y propuesta de un procedimiento para programar por Competencias Básicas. Aula Abierta, 40 (3), 33-46.

OCDE (2006). PISA 2006. Marco de la evaluación. Conocimientos y habilidades en Ciencias, Matemáticas y Lectura. Madrid: Santillana.

OCDE (2009). PISA 2009. Programa para la evaluación internacional de los alumnos. Informe español.

http://iaqse.caib.es/documents/aval2009-10/pisa2009-informe-espanol.pdf

Parlamento Europeo (2006). Recomendación del Parlamento Europeo y del Consejo sobre las competencias clave para el aprendizaje permanente. Diario Oficial de la Unión Europea (2006/962/CE). http://eur-lex.europa.eu/LexUriServ/LexUriServ.do?uri=OJ:L:2006:394:0010:0018:es:pdf

Parrilla, A. (1999). Educational innovations as a school answer to diversity. International Journal of Inclusive Education, 3 (2), 93-110. HTTP://DX.DOI.ORG/10.1080/136031199285066

Pepper, D. (2011). Assessing key competences across the curriculum and Europe. European Journal of Education, 46 (3), 335-353.

Pérez-Pueyo, A. (2012). Análisis de las diferentes propuestas de desarrollo de las competencias básicas. Revista Española de Educación Física y Deportes, 397, 13-36.

Pérez-Pueyo, A. (2013). Programar y evaluar competencias básicas en 15 pasos. Barcelona: Graó.

Pérez-Pueyo, A. y Casanova, P. (2010). La programación de las Competencias Básicas en colegio de infantil y primaria. Una propuesta de secuenciación por ciclos. Madrid: CEP. HTTP://DX.DOI.ORG/10.1111/J.1465-3435.2011.01484.X

Pharo, E. y Bridle, K. (2012). Does interdisciplinarity exist behind the façade of traditional disciplines? A study of natural resource management teaching. Journal of Geography in Higher Education, 36 (1), 65-80. HTTP://DX.DOI.ORG/10.1080/03098265.2011.575127

Polo, I. (2010). La evaluación de las competencias básicas. Revista de la Asociación de Inspectores de Educación de España, 12 (3), 34-45. 
Proyecto Atlántida (2013). Guía para la formación en centros sobre las competencias básicas. http://www.proyectoatlantida.net/.

Real Decreto 126/2014, de 28 de febrero, por el que se establece el currículo básico de la Educación Primaria ( $B O E$ de 1 de marzo de 2014).

Sarramona, J. (2004). Las Competencias Básicas en la Educación Obligatoria. Barcelona: CEAC.

Sebastiani, M.; Blázquez, D. y Borrachina, J. (2009). Concepto y naturaleza de las competencias. En D. Blázquez y M. Sebastiani (Eds.). Enseñar por competencias en Educación Física (pp. 120-132). Barcelona: Inde.

Standage, M.; Duda, J. y Ntoumanis, N. (2003). Predicting motivational regulations in physical education: The interplay between dispositional goal orientations, motivational climate and perceived competence. Journal of Sports Sciences, 21 (8), 631-647. HTTP://DX.DOI.ORG/10.1080/0264041031000101962

Vermeulen, M.; Castelijns, J.; Kools, Q. y Koster, B. (2012). Measuring student teachers' basic psychological need. Journal of Education for Teaching, 38 (4), 453-467. DOI: $10.1080 / 02607476.2012 .688556$.

Zabala, A. y Arnau, L. (2007). Cómo aprender y enseñar competencias. Barcelona: Graó. 